\title{
Rapid EELS and EDS maps of amorphous silicate matrix in Paris and Acfer 094
}

\author{
K. K. OHTAKI*1 ${ }^{* 1}$ J. P. BRADLEY ${ }^{1}$, H. A. ISHII ${ }^{1}$, J.

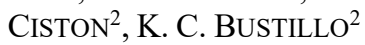

${ }^{1}$ University of Hawai' $i$ at Mānoa, Honolulu, HI

${ }^{2}$ National Center of Electron Microscopy, Molecular Foundry, Lawrence Berkeley National Laboratory, Berkeley, CA

(*correspondence: kohtaki@hawaii.edu)

Materials from cosmically primitive objects like comets and the least-altered asteroids best represent the ancient starting materials from the formation of our Solar System. This study discusses a possible formation mechanism of the amorphous silicate materials commonly present in primitive meteorite matrix. The composition and oxidation state of amorphous silicate materials can be diagnostic of the process(es) they experienced before or during accretion and on the parent body. Meteorites Paris and Acfer 094 were studied since they are believed to have undergone minimal parent body processing, and both contain "GEMS-like" amorphous silicate material similar to that in chondritic porous interplanetary dust particles (CP IDPs), thought to be cometary [1]. TEM specimens were extracted from metal-rich region, using conventional FIB trenching and a hybrid ultramicrotomy-FIB method [2], and mapped with energy dispersive X-ray spectroscopy (EDS) and electron energy loss spectroscopy (EELS). Fe oxidation state distributions, chemical shifts, and core-loss fine-structures were measured over 0-3000 eV using the Continuum EEL spectrometer.

Several indicators of aqueous alteration and weathering of minerals were observed around forsterite crystals in the meterites; e.g. "fuzzy" crystal edges, nanocrystals with $\mathrm{Mg}$ depletion, increasing Fe concentration approaching Fe-sulfide grains, and $\mathrm{O}-\mathrm{K}$ pre-edge in the amorphous silicate. The formation mechanism of amorphous silicate in primitive meteorite matrices is still under debate, and some suggest direct condensation in solar nebula prior to accretion $[3,4]$ but the EELS oxidation map results combined with EDS and diffraction data in this study suggest an alternative formation route for meteorite amorphous silicate matrix: amorphization of crystalline silicates and $\mathrm{Fe}^{3+}$ infiltration of the amorphous silicate from oxidative decomposition of $\mathrm{Fe}$-metal and $\mathrm{Fe}^{2+}$ rich sulfides during parent body aqueous alteration.

References: [1] Leroux H. et al. (2015), GCA, 170, 247-265, [2] Ohtaki K. K. et al. (2019), Microsc. Microanal., in press [3] Brearley A. J. et al. (1995), GCA, 59, 4307-4316 [4] Abreu N. M. \& Brearley A. J. (2010) GCA, 74, 1146-1171. 